\title{
Reproducible and Sensitive Plasmonic Sensing Platforms Based on Au-Nanoparticle-Internalized Nanodimpled Substrates
}

\author{
Hajun Dang, Sung-Gyu Park, Yixuan Wu, Namhyun Choi, Jun-Yeong Yang, \\ Seunghun Lee, Sang-Woo Joo,* Lingxin Chen, * and Jaebum Choo*
}

Electromagnetic enhancement effects through localized surface plasmon resonance considerably amplify the intensity of incident light when molecules are positioned in the vicinity of miniscule nanogaps. The aggregation of plasmonic nanoparticles synthesized using bottom-up methods has been extensively used to generate hot spots in solutions. These methods assist in obtaining non-periodic plasmonic signals, because the realization of uniform nanogaps through particle aggregation is difficult. Nanostructured substrates with gaps of 20-100 nm have also been fabricated using the top-down approach. However, the fabrication of smaller nanogap templates using these methods is difficult owing to high costs and low throughput. Therefore, a nanodimple array internalized with AuNPs is developed in this study to mitigate the challenges encountered in the bottom-up and top-down approaches. Precise nanogaps are generated by regularly internalizing AuNPs in the cavities of nanodimples through DNA hybridization. Simulations of the electric field distribution indicate that the incorporation of $80 \mathrm{~nm}$-sized AuNPs into a curved nanodimpled Au substrate generate high-density volumetric hot spots within a detection volume, and result in a high plasmonic enhancement factor of $8.25 \times 10^{7}$. The tremendous potential of the proposed plasmonic platform as an SERS-based biomedical diagnostic device is also verified.

technologies, such as e-beam lithography, focused ion beam lithography, and photolithography, have been extensively used to prepare organized nanostructured substrates with gaps sized 20-100 nm. ${ }^{[4-6]}$ Despite such regularities, these methods encounter limitations in fabricating templates with significantly smaller nanogaps owing to high costs and inefficient outputs. Nanometer gap structures can also be generated via the bottom-up self-assembly of Au nanoparticles (AuNPs) through electrostatic interactions or deoxyribonucleic acid (DNA) hybridization. ${ }^{[7-14]}$ However, despite numerous studies on the bottomup assembly of metal nanoparticles, these systems have yielded suspended structures that have complicated the precise evaluation of the enhancements.

To overcome the aforementioned problem, a new nanoplasmonic platform, which comprises AuNPs regularly arranged on a nanopore substrate, was recently developed by our group; ${ }^{[15]}$ therein, a new ultrasonic technique was

\section{Introduction}

Electromagnetic enhancements through plasmon resonance can significantly intensify light that is incident on localized cavities of small nanogaps. ${ }^{[1-3]}$ Several nanostructured substrates have been fabricated using the top-down approach to amplify optical plasmon resonance. Semiconductor processing developed for incorporating monomeric AuNP arrays into anodized aluminum nanopores by a direct route. However, the gap had to be as large as $12 \mathrm{~nm}$ owing to the fixed metal-template substrates, resulting in an enhancement factor (EF) of only $2.5 \times 10^{4}$. As EFs are substantially dependent on the nanogap distances, the efficient assembly of smaller nanogaps should be beneficial for realizing additional practical applications.
H. Dang, Y. Wu, N. Choi, J. Choo

Department of Chemistry

Chung-Ang University

Seoul 06974, South Korea

E-mail: jbchoo@cau.ac.kr

S.-G. Park, J.-Y. Yang, S. Lee

Nano-Bio Convergence Department

Korea Institute of Materials Science (KIMS)

Changwon 51508, South Korea

The ORCID identification number(s) for the author(s) of this article can be found under https://doi.org/10.1002/adfm.202105703.

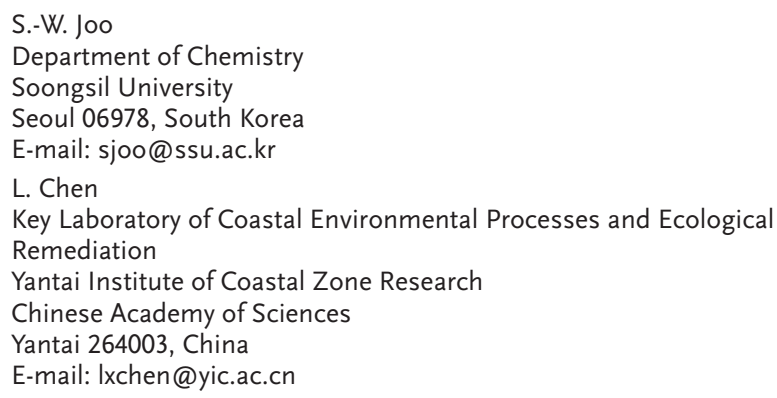

E-mail: Ixchen@yic.ac.cn

DOI: 10.1002/adfm.202105703 
A new method, inspired by the regular assembly of AuNPs in nanopore templates, was developed in the present study for preparing effective surface-enhanced Raman scattering (SERS) substrates sandwiched between the AuNPs and curved cavities of a nanodimpled Au substrate. ${ }^{[16,17]}$ To implement this platform, a Au layer was deposited on a nanodimple-patterned polyethylene naphthalate (PEN) substrate via thermal evaporation to prepare a plasmonic nanodimpled Au substrate. Subsequently, the AuNPs were inserted into the curved cavities of the nanodimpled $\mathrm{Au}$ substrate using the following two methods: electrostatic interactions using 4-aminothiophenol (ATP) and DNA hybridization. After inserting the AuNPs in the cavities of the nanodimples using ATPs and DNAs, the corresponding nanogaps between the AuNPs and curved cavity surface were estimated to be $\approx 1$ and $4 \mathrm{~nm}$, respectively. Finite-difference time-domain (FDTD) simulations were employed to confirm that the nanogaps effectively generated the electromagnetic field of the array. Simulations of the electric field distribution demonstrated that the incorporation of $80 \mathrm{~nm}$-sized AuNPs into the 3D curved cavities of the nanodimpled $\mathrm{Au}$ substrate yielded high-density volumetric hot spots within a detection volume, resulting in a high SERS EF of $10^{8}$.

Finally, high-sensitivity detection experiments were performed on COVID-19 bridge DNAs to investigate the possibility of applying the designed $\mathrm{Au}$ nanoplasmonic platform as a biomedical diagnostic device. The reverse transcriptionpolymerase chain reaction (RT-PCR) technique is used as a standard diagnostic method for COVID-19 as this method has high diagnostic sensitivity and specificity. ${ }^{[18-20]}$ Nonetheless, the concentration of the target DNA must be amplified through a 30-40 thermo-cycling process due to the detection limit of the fluorescence detection method in RT-PCR, requiring a diagnosis time of at least 3-4 h. To address this problem, we recently developed a SERS-PCR method using magnetic beads. ${ }^{[21]}$ The enhanced detection capability of the AuNP-internalized nanodimpled substrate was investigated in the present study for further decreasing the number of detectable thermocycling steps. The detection sensitivity determined using a SERS-based assay with magnetic beads was compared with that obtained from an assay with the nanodimpled Au substrate. The RdRp and E-bridge probe genes were used as target DNAs in the SERS-PCR experiments; these are representative targets used for detecting COVID-19. The high-density volumetric hotspot generation capability of the designed AuNP-internalized nanodimpled platform was experimentally verified and its applicability with respect to the SERS-PCR was evaluated. Therefore, this technology is expected as a new platform technology that can shorten the PCR measurement time.

\section{Results and Discussion}

\subsection{Fabrication of Nanodimpled Substrates and Internalization of AuNPs}

The construction of a robust and reproducible SERS detection platform was attempted herein using AuNP-inserted nanodimpled substrates. The fabrication of Au-nanodimpled plasmonic substrates and the internalization of AuNPs into their curved cavities are depicted in Figure 1A. First, a nanodimple-patterned PEN substrate was fabricated using oxygen ion beam sputtering. Subsequently, a $150 \mathrm{~nm}$-thick Au layer was directly deposited onto the nanodimpled PEN substrate via thermal evaporation. ${ }^{[16,17]}$ The average diameter of cavities in the substrate was estimated to be $107 \mathrm{~nm}$ (Figure S1, Supporting Information). Two different binding agents were employed to optimize the plasmonic coupling between the AuNPs and the curved cavities. AuNPs were inserted into the cavities of the nanodimpled substrate through two different methods, as shown in Figure 1B; one involved electrostatic interactions using ATP and the other involved DNA hybridization. In the method involving electrostatic interactions, the thiol group at one end of ATP binds with the surface of the Au cavity through an $-\mathrm{SH}$ covalent bond. The amine group at the other end can attach onto the negatively charged AuNPs through electrostatic interactions [Figure 1C(i)]. In DNA hybridization, capture DNAs (5'-Cy5-TTG AGG AGA ATT TTT TTT TT- $\left.\left(\mathrm{CH}_{2}\right)_{3}-\mathrm{SH}-3^{\prime}\right)$ were immobilized on the surface of the nanodimpled $\mathrm{Au}$ substrates. Complementary DNA $\left(5^{\prime}-\right.$ TTC TCC TCA ATT TTT TTT TT- $\left.\left(\mathrm{CH}_{2}\right)_{3}-\mathrm{HS}-3^{\prime}\right)$-attached AuNPs were internalized into the cavities through DNA hybridization [Figure 1C(ii)].

\subsection{Effects of the Substrate Shape on Plasmonic Coupling with Nanoparticles}

Huang et al. previously reported that a AuNP induces a significantly stronger local field enhancement of adsorbed molecules in a cavity compared to that on a flat film. ${ }^{[22]}$ Therefore, SERS signals of AuNPs in the cavities of the nanodimpled substrate were measured to experimentally confirm the electromagnetic enhancement in a large-area cavity platform. The surface plasmon coupling effect of AuNPs loaded on a flat Au film was also evaluated to compare the SERS enhancement effects of AuNPs on different Au substrates. In both cases, AuNPs were immobilized onto the substrates through electrostatic interactions using ATP (Figure 2A). The corresponding transmission electron microscopy (TEM) images show that the $80 \mathrm{~nm}$-sized AuNP is separated from the surface of the Au substrate by a $1 \mathrm{~nm}$-sized ATP spacer (yellow boxes on the TEM images in Figure 2A).

FDTD simulations were also performed to elucidate the plasmonic coupling effects between the AuNPs and the substrate. Figure 2B(i),(ii) show the electric field distributions of a AuNP in a cavity of the nanodimpled substrate and on a flat film, respectively. Linearly polarized light with a wavelength of $633 \mathrm{~nm}$ was directly illuminated onto the AuNP on the two different substrates. The nanogap between the AuNP and the surface was set to $1 \mathrm{~nm}$ based on the TEM results. Simulation results of the $x-z$ and $x-y$ sections of the AuNP in the cavity of the nanodimpled substrate and on the flat film, respectively, are shown in Figure 2B. Figure 2B(ii) indicates that the simulated electric field of a AuNP on a flat film is highly localized at the AuNP-surface junction at a miniscule spot. The electric field evaluated for the AuNP in the cavity of the nanodimpled substrate is localized at a larger curved junction area between the AuNP and the cavity [Figure 2B(i)]. The point-spot coupling between the AuNP and the cavity is weaker than that of the flat 
(a)
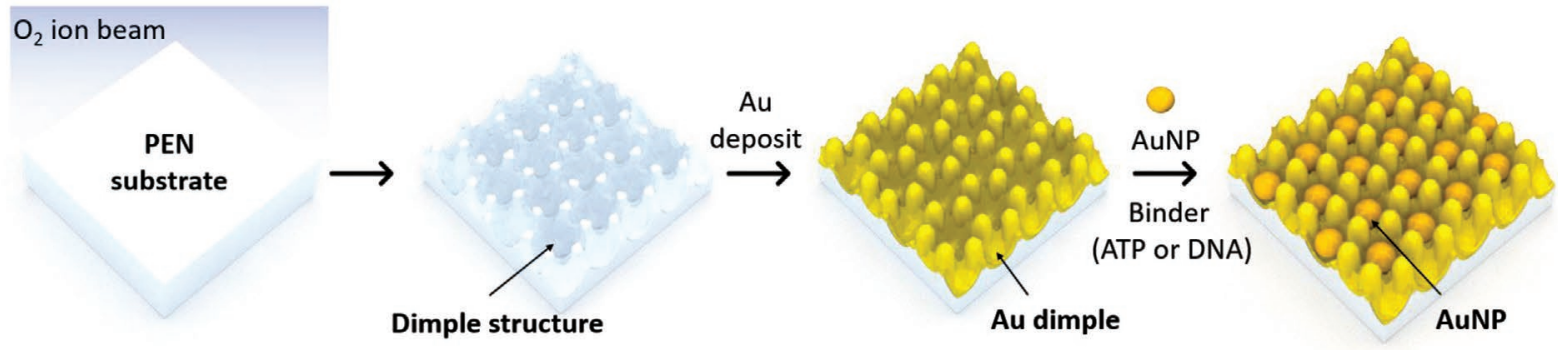

(b)
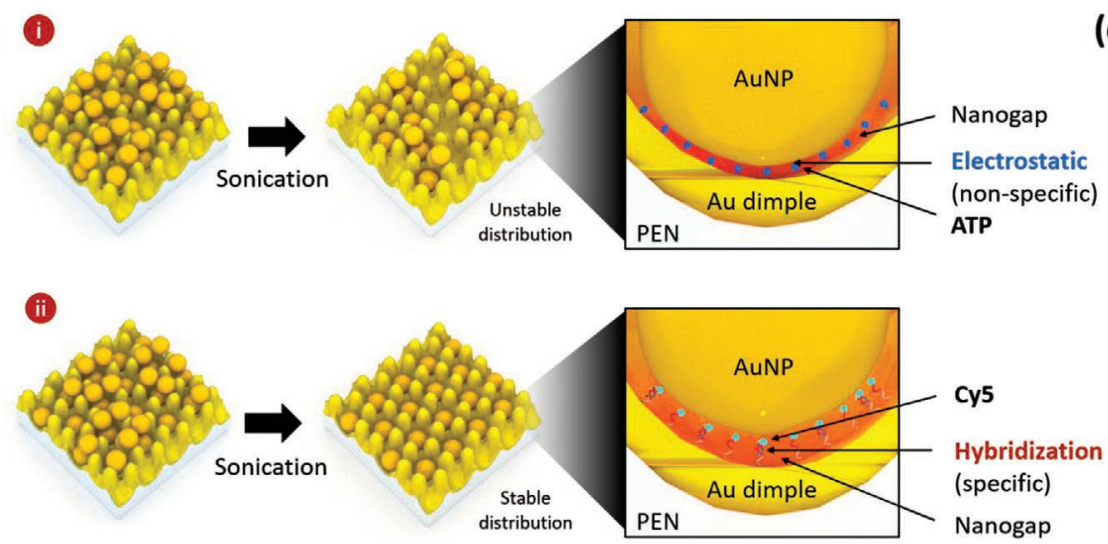

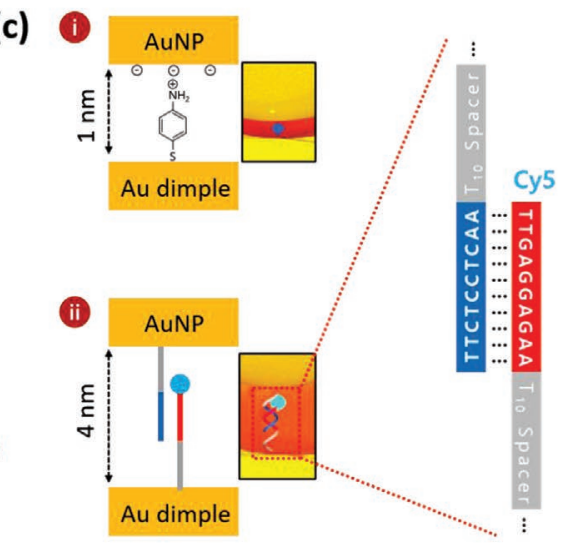

Figure 1. Schematic of a) fabrication of a nanodimpled Au substrate and internalization of AuNPs. b) Two methods employed to internalize AuNPs in the cavities of the nanodimpled substrate through i) electrostatic interactions and ii) DNA hybridization. c) Binding interactions between a AuNP and a nanodimpled Au cavity based on i) electrostatic interactions and ii) DNA hybridization.

film. However, the overall area of the high-density hotspot in the cavity is considerably larger than that on the flat film, as evidenced by the corresponding $x-y$ section images in Figure 2B.

The SERS mapping images in Figure $2 \mathrm{C}$ demonstrate that the AuNPs in the cavities of the nanodimpled substrate show a more substantial Raman signal enhancement [Figure 2C(i)] than that on the flat film [Figure 2C(ii)]. The variations in the most intense Raman peak of ATP at $1080 \mathrm{~cm}^{-1}$ were used to obtain the Raman mapping images. Figure 2D compares the averaged Raman spectra obtained using 2500 pixels of the corresponding Raman mapping images in Figure 2C. The uniform bright-yellow color of the mapping image and the strong Raman peak intensities of AuNPs in the cavities indicate substantial plasmonic coupling of the localized surface plasmon of AuNPs with the surface polariton of the cavities of the nanodimpled substrate.

\subsection{Effects of Binding Agent on the Uniform Internalization of Nanoparticles}

DNA hybridization is known to result in more specific and robust interactions between AuNPs and $\mathrm{Au}$ cavities than electrostatic interactions. ${ }^{[23,24]}$ The effectiveness of these selfassembly methods for the systematic insertion of AuNPs into the nanodimpled-substrate cavities was investigated. Figure 3 shows AuNP-internalized nanodimpled substrates fabricated via electrostatic interactions and DNA hybridization; the corresponding top-view and cross-sectional scanning electron microscopy (SEM) images are also shown. Non-specific AuNPs were removed from the substrate via sonication for $20 \mathrm{~s}$. SEM images were acquired before and after the sonication of samples obtained via both, electrostatic interactions and DNA hybridization, to compare the effects of the binding agents and their efficiencies with respect to the uniform distribution (Figure S2A, Supporting Information). Non-specific AuNPs were more easily removed via sonication through the method employing electrostatic interactions, owing to weak interaction forces [Figure S2C(i), Supporting Information]. However, a greater number of AuNPs remained in the cavities owing to the relatively stronger interactions realized via DNA hybridization [Figure S2C(ii), Supporting Information]. Figure 4A shows SEM images and the corresponding SERS mapping images [ATP at $1080 \mathrm{~cm}^{-1}$ and Cy5-labeled DNA hybridization at $1595 \mathrm{~cm}^{-1}$ ] of $80 \mathrm{~nm}$-sized AuNP-internalized nanodimpled substrates. As shown in the SEM images, the AuNPs distribute more uniformly in the cavities through DNA hybridization [Figure 4a(ii), left]. Consequently, the corresponding Raman spectra of all the pixels in the Raman mapping images show a significantly better uniformity than those obtained via electrostatic interactions using ATP (Figure 4B). The relative standard deviations (RSDs) of 2500 Raman intensity distributions are estimated to be $29.87 \%$ and $8.24 \%$ for samples prepared via electrostatic interactions and DNA hybridization, respectively (Figure 4C). Therefore, the Raman spectral data reveal that the AuNPs are more uniformly distributed in the cavities through 
(a)

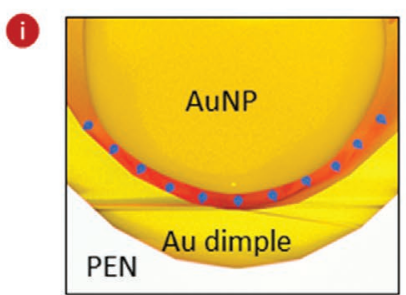

(1)

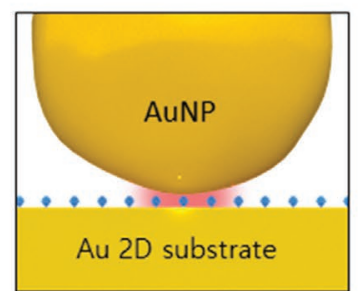

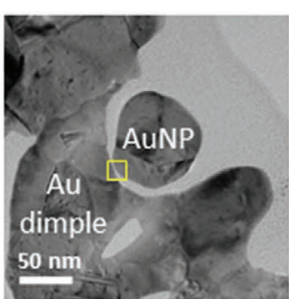

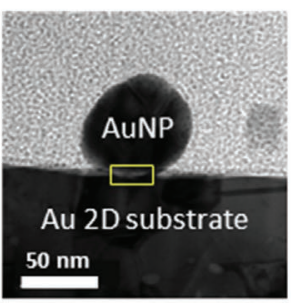

(b)

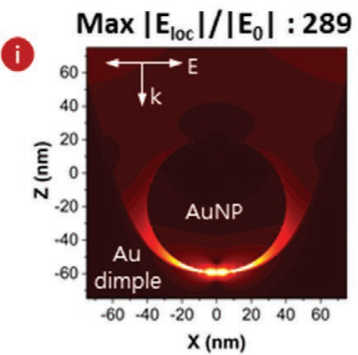

(ii) $\operatorname{Max}\left|E_{10 c}\right| /\left|E_{0}\right|: 112.5$

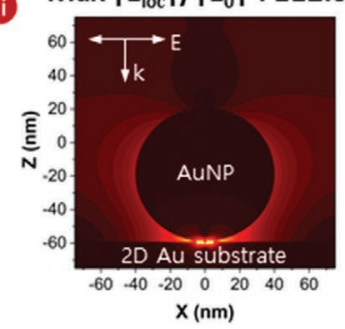

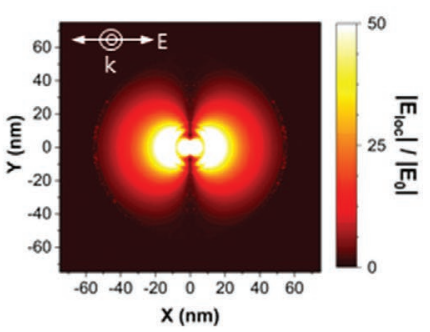

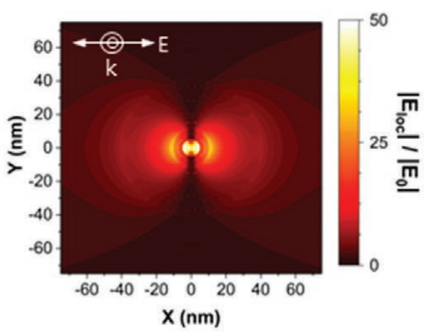

(c)

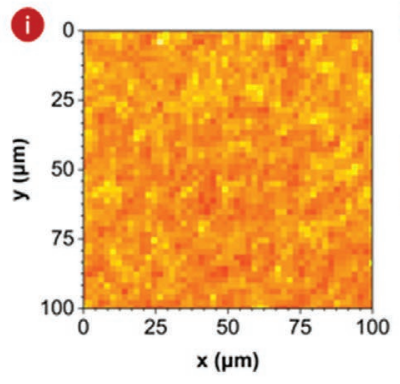

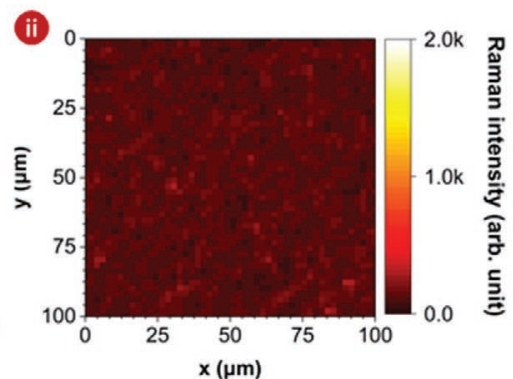

(d)

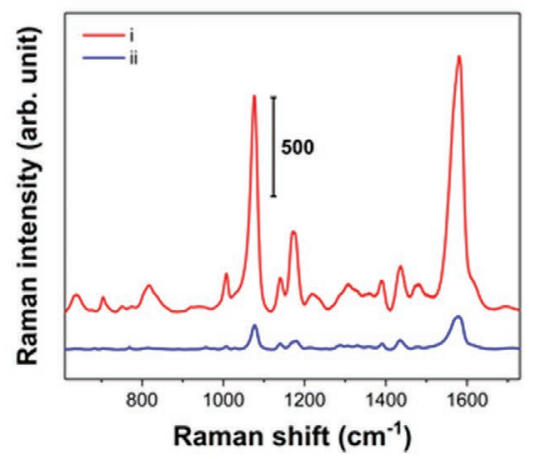

Figure 2. Comparison of SERS signal enhancements with varying plasmonic substrate conditions. a) Schematics and corresponding cross-sectional TEM images of $80 \mathrm{~nm}$-sized AuNPs loaded in a i) nanodimpled Au cavity and on a ii) 2D planar Au substrate using ATP. An $\approx 1 \mathrm{~nm}$-sized plasmonic nanogap exists between the dimpled cavities and the AuNPs (yellow rectangles). b) FDTD simulation of the electric field distribution in the i) cavity and on the ii) 2D Au substrate loaded with $80 \mathrm{~nm}$-sized AuNPs [gap size: $1 \mathrm{~nm} ; x-z$ section (left) and $x-y$ section (right)]. The scale bars on the right show the color-decoding scheme for different electromagnetic field intensities. c) SERS mapping images of ATP on $80 \mathrm{~nm}$-sized AuNP-loaded i) nanodimpled and ii) $2 \mathrm{D}$ substrates. Images were obtained using the peak intensity at $1080 \mathrm{~cm}^{-1}$ and scanning an area of $100 \times 100 \mu \mathrm{m}^{2}$ with a $2 \mu \mathrm{m}$ interval. d) Average of the Raman spectra obtained using all 2500 pixels of the corresponding Raman mapping images shown in Figure $2 \mathrm{C}$ (i) and (ii).

DNA hybridization than through electrostatic interactions. The large cross-section of the Raman reporter, Cy5, and the uniform distribution of AuNPs through DNA hybridization, result in intense SERS signals. Furthermore, the Cy5 reporter has a maximum absorption wavelength at $648 \mathrm{~nm}$, which is close to the laser wavelength at $633 \mathrm{~nm}$. When the wavelength of the laser beam is comparable to the electronic transition wavelength of a Raman reporter, significant electromagnetic enhancement is observed due to resonance effects.

The insertions of $80 \mathrm{~nm}$-sized AuNPs into nanodimpled $\mathrm{Au}$ substrates using the two different binding methods were compared over a large area $\left(10 \times 10 \mu \mathrm{m}^{2}\right)$ to obtain more reliable results on the uniform distribution of $\mathrm{Au}$ particles (Figure S3, Supporting Information). Figure S3A, Supporting Information, shows SEM images of the AuNP-inserted nanodimpled cavities obtained through electrostatic interactions (ATP) and DNA hybridization (DNA-Cy5) after sonication. The DNA hybridization complex formed in wet conditions has a length of $\approx 10-15 \mathrm{~nm}$, but is reduced to less than $5 \mathrm{~nm}$ in dry conditions. This reduction in DNA length in dry conditions has also been reported in previous papers. ${ }^{[12,25]}$ As shown in Figure 3B(ii), the nanogap between AuNP and the cavity is estimated to be $\approx 4 \mathrm{~nm}$, and this is much shorter than the distance in wet conditions. The average numbers of AuNPs in the $1 \times 1 \mu \mathrm{m}^{2}$ cavity were estimated to be 19 and 27 for the ATP method and DNA hybridization, respectively. As shown in Figure S3B, Supporting Information, the corresponding RSDs were calculated to be $20.02 \%$ and $10.02 \%$ for the nanodimpled Au substrates with an area of $10 \times 10 \mu \mathrm{m}^{2}$ obtained via electrostatic interactions and DNA hybridization, respectively. Therefore, the uniform distribution of AuNPs can be also achieved on a nanodimpled substrate with a large area through DNA hybridization.

\subsection{Effects of Particle Size on Plasmonic Coupling with Nanodimpled Substrates}

Three different sizes of AuNPs (20, 50, and $80 \mathrm{~nm})$ were inserted into the cavities, and their SERS signals were measured to optimize the nanoparticle size for facilitating plasmonic 
(a)

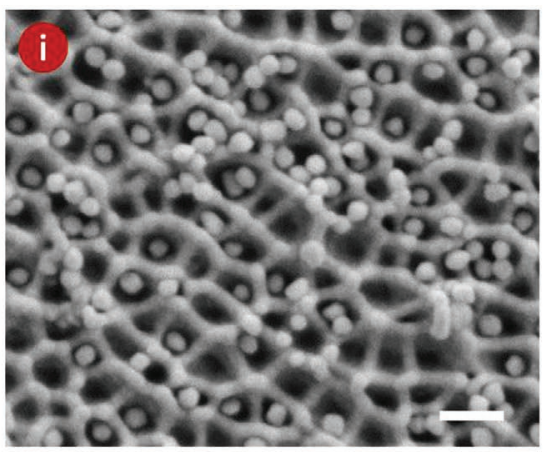

(b)

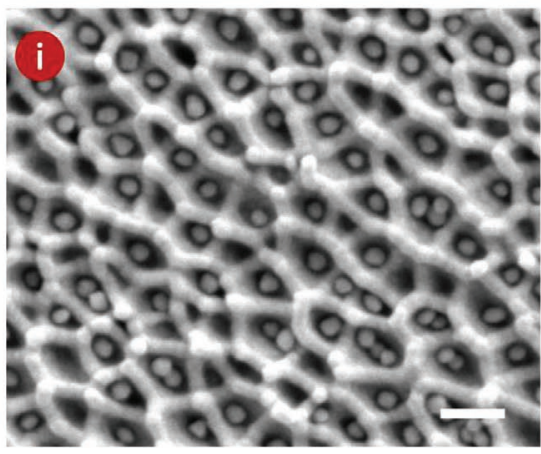

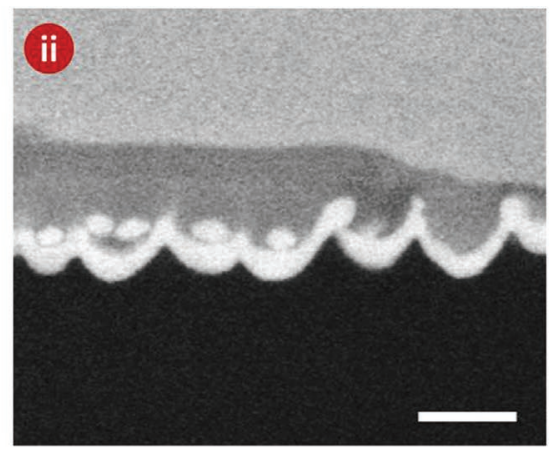

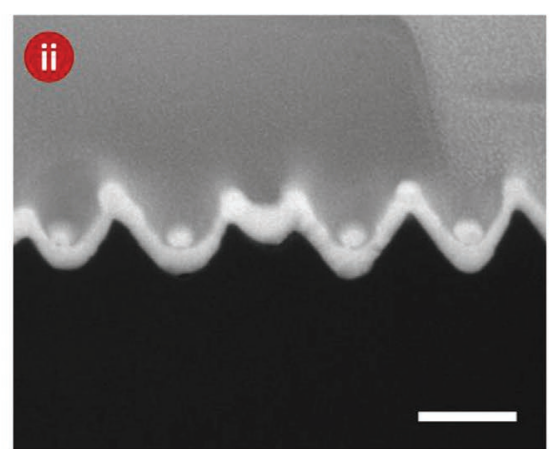

iii

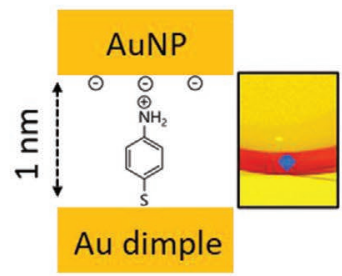

iii

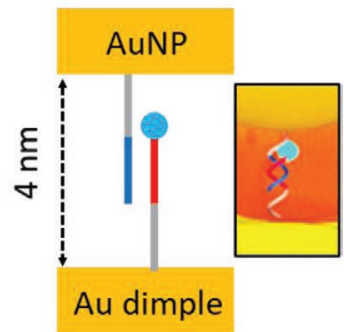

Figure 3. SEM images and schematics of AuNP-internalized nanodimpled Au substrates fabricated through a) electrostatic interactions and b) DNA hybridization. i) Top-view and ii) cross-sectional SEM images (scale bars: $200 \mathrm{~nm}$ ). iii) Schematics of the binding interactions between a AuNP and a nanodimpled Au cavity.

coupling between the AuNPs and the dimpled cavities. The AuNPs $(20,50$, and $80 \mathrm{~nm})$ were prepared using the seed growth method. ${ }^{[27]}$ Figure S4A, Supporting Information, shows TEM images of the i) 20, ii) 50, and iii) $80 \mathrm{~nm}$-sized AuNPs. Figure S4B,C, Supporting Information, shows the corresponding UV-vis spectra and dynamic light scattering (DLS) distribution curves. The increase in particle size shifts the UVvis absorption maximum and the size distribution in DLS to longer wavelengths. DNA-conjugated AuNPs (TTC TCC TCA ATT TTT TTT TT- $(\mathrm{CH} 2)_{3}-\mathrm{S}-\mathrm{Au}$ ) were identified using UVvis spectra, DLS, and energy-dispersive X-ray spectroscopy (EDS) elemental mapping analysis, as shown in Figure S5, Supporting Information. The UV-vis spectra in Figure S5A, Supporting Information, show slight red shifts for AuNPs with DNAs attached to them. The DLS distributions in Figure S5B, Supporting Information, indicate that the DNA attachment also increases the AuNP size. Figure S5C, Supporting Information, shows the EDS mapping images of gold (yellow) and phosphorous (red) obtained from the DNA backbones, with the fourth image representing an overlay. The distribution of the redcolored phosphorous in the mapping images confirms that the detection DNAs have been successfully attached to the AuNP surfaces.

The capture DNAs were immobilized on $5 \times 5 \mathrm{~mm}^{2}$ dimpled $\mathrm{Au}$ substrates, and three different sizes of complementary DNA-attached AuNPs were internalized in the cavities. The AuNPs were attached to the cavity through DNA hybridization. Figure 5A shows SEM images of the nanodimpled substrate, and of substrates internalized with 20, 50, and $80 \mathrm{~nm}$-sized AuNPs. The corresponding SERS mapping images are shown in Figure 5B. The variation in the Raman peak intensity at
$1595 \mathrm{~cm}^{-1}$ was used to obtain Raman mapping images with $2 \times$ $2 \mu \mathrm{m}^{2}$ mapping steps over an area of $100 \times 100 \mu \mathrm{m}^{2}$. Figure $5 \mathrm{C}$ shows the averaged Raman spectra obtained using 2500 pixels of the corresponding Raman mapping images shown in Figure 5B. The Raman signal intensity is enhanced by 1.2 (20 nm), $4.0(50 \mathrm{~nm})$, and 9.0 times $(80 \mathrm{~nm})$ compared with that of the Raman spectrum of Cy5 in the empty Au cavity. The corresponding RSDs are estimated to be $24.80 \%, 14.85 \%$, and $8.24 \%$, respectively.

This indicates that the $80 \mathrm{~nm}$-sized AuNPs exhibit a more substantial plasmonic coupling effect than the $50 \mathrm{~nm}$-sized AuNP. Therefore, the Raman measurements confirm that the $80 \mathrm{~nm}$-sized AuNP-internalized nanodimpled substrate exhibits the strongest SERS signal with high reproducibility.

\subsection{Potential Feasibility of AuNP-Inserted Nanodimpled Substrates as SERS-PCR Platforms}

The RT-PCR is used for quantitative analysis of the target DNA. However, this method requires a long detection time because of the insufficient sensitivity of the fluorescence detection method for detecting low concentrations of DNA; consequently, the target DNA is amplified through lengthy thermocycling steps. An SERS-based PCR assay platform that enabled the rapid and sensitive detection of a target gene at low concentrations was recently developed by our group; ${ }^{[21]}$ therein, the number of thermocycling steps required to detect the target DNA was drastically reduced from 15 to 5 using the magnetic-bead-based SERS-PCR platform instead of the conventional fluorescence RT-PCR method. However, the amplification-free detection 
(a)

(b)
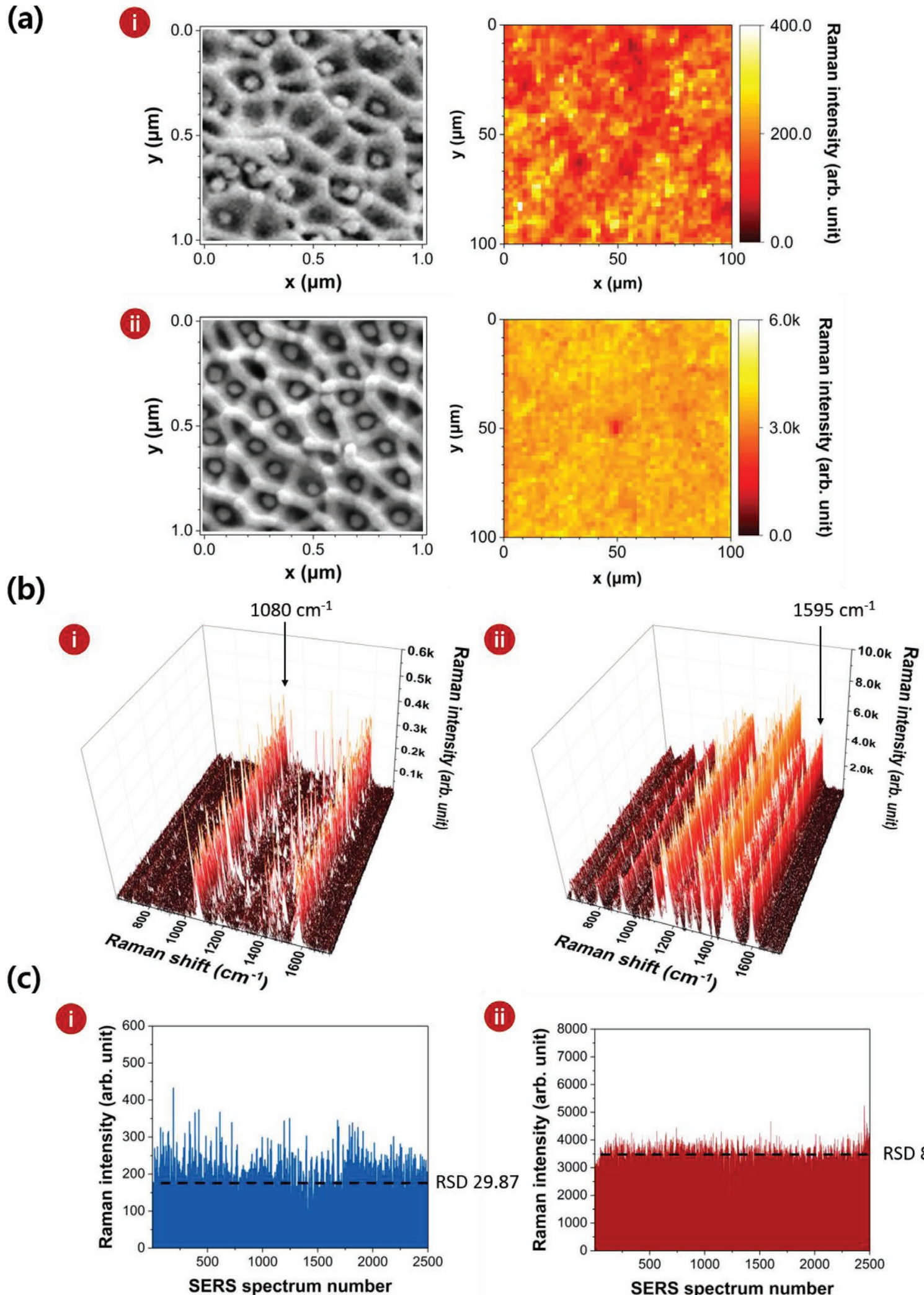

(ii)

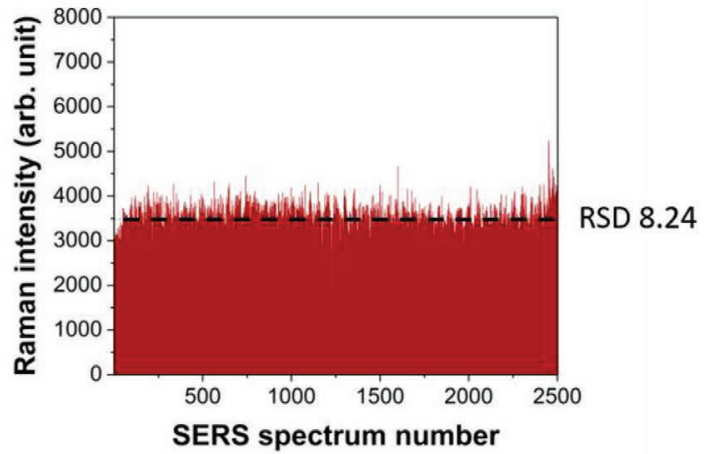

Figure 4. a) SEM (left) and Raman mapping (right) images of $80 \mathrm{~nm}$-sized AuNP-internalized nanodimpled Au cavities obtained through i) electrostatic interactions and ii) DNA hybridization after sonification. SERS mapping images correspond to i) $1080 \mathrm{~cm}^{-1}$ (ATP) and ii) $1595 \mathrm{~cm}^{-1}$ (DNA-Cy5). b) SERS spectra of i) ATP and ii) Cy5 after sonication. c) Variations in the distribution of Raman intensities at i) $1080 \mathrm{~cm}^{-1}$ (ATP) and ii) $1595 \mathrm{~cm}^{-1}$ (DNA-Cy5). 
(a)

(b)
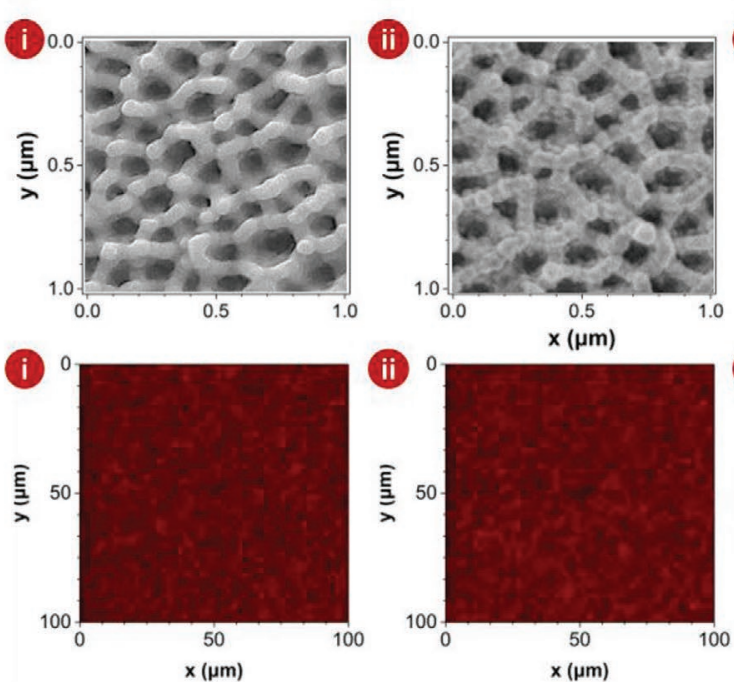

(c)
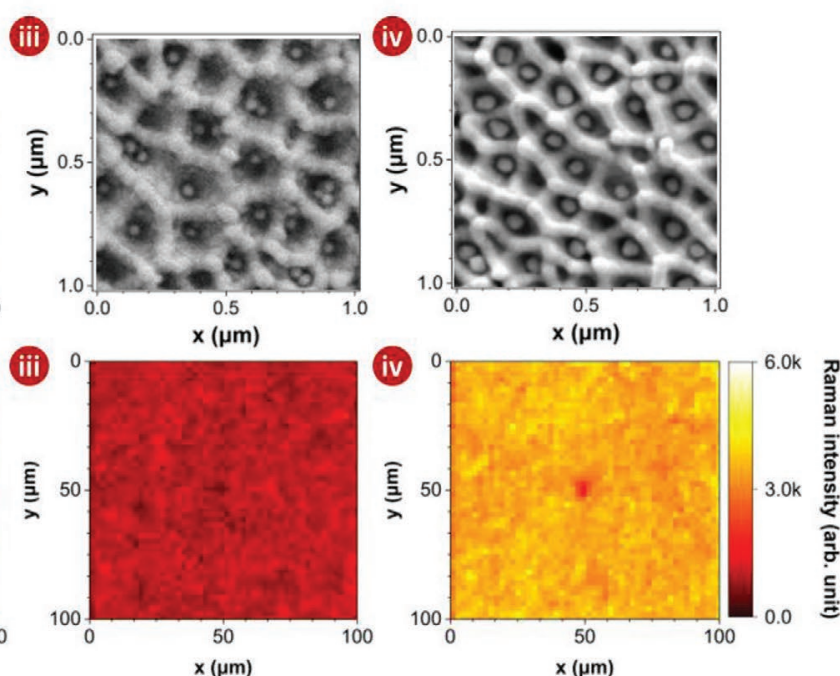

$1595 \mathrm{~cm}^{-1}$

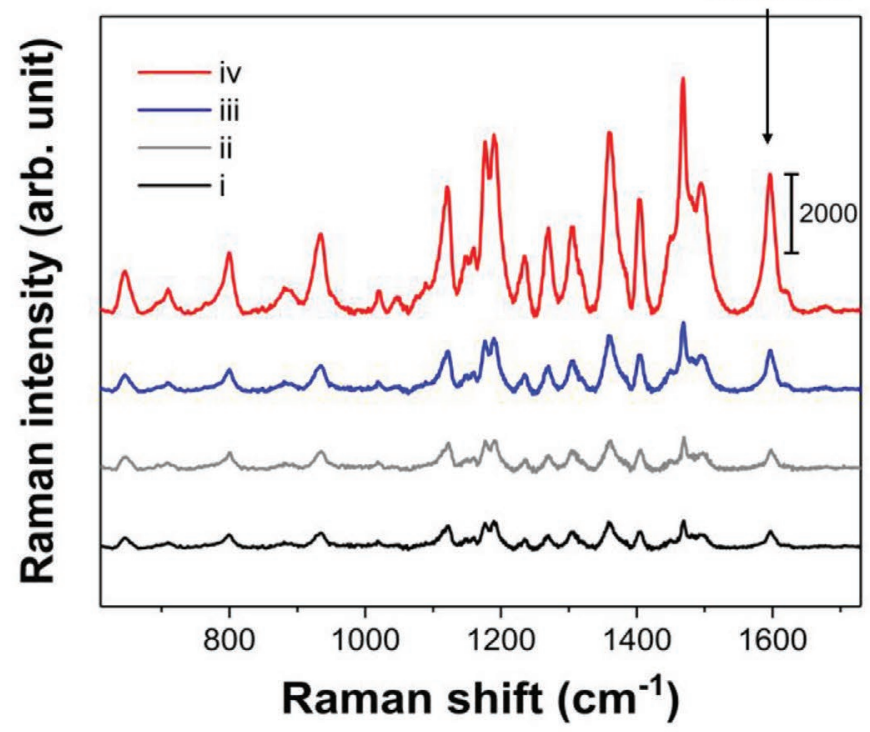

Figure 5. Evaluation of SERS signal amplification for different sizes of AuNP-internalized nanodimpled substrates. a) SEM and b) Raman mapping images of the i) nanodimpled Au substrate and nanodimpled substrates internalized with ii) 20, iii) 50, and iv) 80 nm-sized AuNPs. DNA hybridization is used to internalize the AuNPs. The diffraction-limited focal spot size of the laser beam in the SEM images was estimated to be $\approx 0.97 \mu \mathrm{m}$. Raman images acquired by mapping the Raman peak intensity at $1595 \mathrm{~cm}^{-1}$ and by scanning a range of $100 \times 100 \mu \mathrm{m}^{2}$ with a $2 \mu \mathrm{m}$ interval. c) Averaged SERS spectra obtained using all 2500 pixels of the corresponding Raman mapping images shown in b).

of target DNAs requires the development of a more sensitive SERS-based assay platform.

Accordingly, the AuNP-inserted nanodimpled system designed in the present study was investigated as a potential diagnostic platform for the amplification-free detection of two SARS-CoV-2 target genes (RdRp and E). A label-free bridge probe was employed in the SERS-PCR instead of the fluorescence dye-labeled TaqMan probe used in the RT-PCR. The SERS-based assays for the RdRp and E genes were performed using magnetic beads and nanodimpled substrates to compare their detection sensitivities. Base sequences of COVID-19 target bridge DNAs, and corresponding capture and detection probe DNAs used for magnetic-bead-based and Au-nanodimple array-based SERS assays were displayed in Table S1, Supporting
Information. A capture-DNA-target-DNA-detection-DNA complex was formed between the surface of the magnetic bead and the AuNP in the magnetic-bead-based assay [Figure 6A(i)]. However, the complex was formed between the AuNP and the dimpled cavity in the nanodimple-based assay [Figure 6B(i)]. Figure S6a,b, Supporting Information, shows the changes in the Raman spectra of the SERS-based assays using magnetic beads and nanodimpled substrates in the $10^{-5}-10^{2} \mathrm{~nm}$ range of the RdRp and E genes. The corresponding calibration curves for the RdRp and E genes are shown in Figure 6. Malachite green isothiocyanate (MGITC) was used as the Raman reporter, and the changes in the intensity of the characteristic Raman peak at $1615 \mathrm{~cm}^{-1}$ were monitored. The limits of detection (LODs) of the SERS-based assays using magnetic beads and the 
(a)

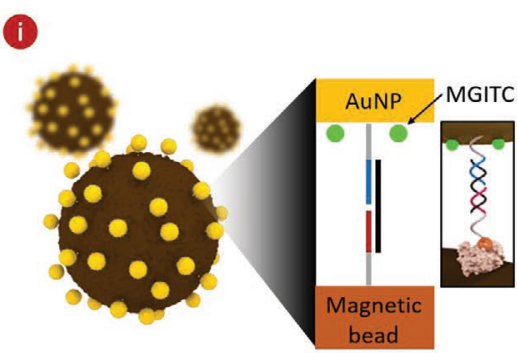

(b)

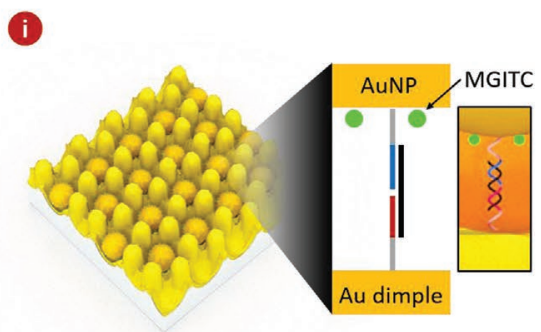

ii

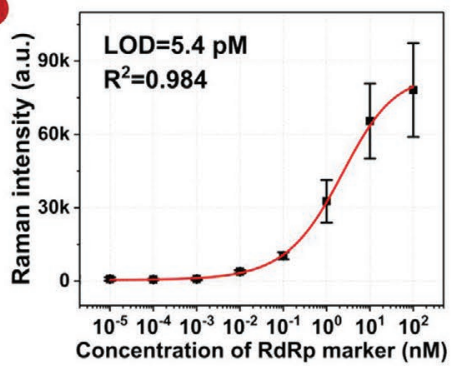

(ii)

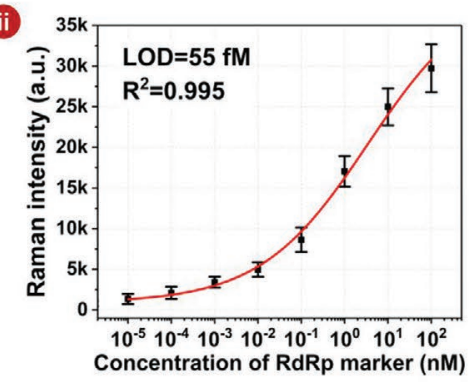

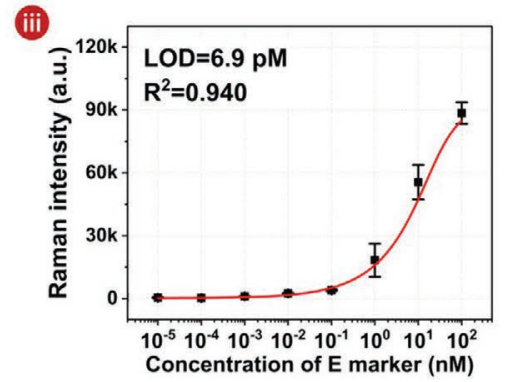

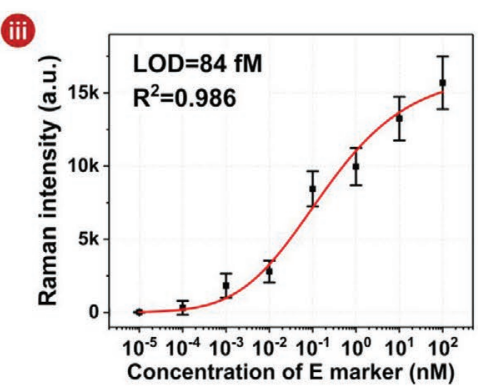

Figure 6. i) Schematics of SERS-based assays using a) magnetic beads and b) nanodimpled Au substrates. Detection and capture DNAs hybridize with a target bridge DNA in the form of a sandwich. Calibration curves obtained using SERS-based assays of two COVID-19 target bridge DNAs, ii) RdRp and iii) E.

nanodimpled substrates for RdRp were evaluated to be 5.4 pм and $55 \mathrm{fm}$, respectively; the corresponding LODs determined for the E gene were $6.9 \mathrm{pm}$ and $84 \mathrm{fm}$. The SERS-based assay with the nanodimpled platform is noted to improve the LOD by $\approx 100$ times compared to that obtained using magnetic beads. Therefore, the SERS-based assay platform using nanodimpled substrates shows tremendous promise for use as an SERS-PCR platform in highly sensitive DNA analysis. The SERS EF was also determined by comparing the Raman peak intensity of $1.25 \times 10^{-8} \mathrm{M}$ MGITC in the AuNP-internalized nanodimpled substrates with that of $5.00 \times 10^{-3} \mathrm{M}$ MGITC in aqueous solution. The EFs were evaluated to be $8.25 \times 10^{7}$.

\section{Conclusions}

In this study, a novel SERS detection platform based on a nanodimpled $\mathrm{Au}$ substrate with $80 \mathrm{~nm}$-sized AuNPs uniformly inserted into its cavities was developed using DNA hybridization. The nanogaps between the AuNPs and curved cavities of the nanodimpled Au substrates formed high-density volumetric hot spots and exhibited an extremely high SERS EF of $8.25 \times 10^{7}$. The FDTD simulations indicated that the AuNP-internalized nanodimpled substrate exhibited a high EF because of the localization of the electric field in the high-density volumetric hotspot area between the AuNPs and curved cavities. The effects of the shape of the substrate, binding agent, and particle size were systematically explored to optimize the localized surface plasmon effect. This system can be applied to the SERS-PCR, and can drastically reduce the number of thermocyclic steps involved in conventional RT-PCR. The assay results obtained using the magnetic-bead-based SERS method were compared with those obtained using the AuNP-internalized nanodimpled substrate for two COVID-19 DNA biomarkers; the LOD of the latter decreased by $\approx 100$ times. This innovative SERS-based assay platform can be employed in high-speed molecular diagnostic technologies based on high-sensitivity gene analysis.

\section{Experimental Section}

Materials: Gold(III) chloride trihydrate $\left(\mathrm{HAuCl}_{4} \cdot 3 \mathrm{H}_{2} \mathrm{O}\right)$, sodium citrate tribasic dihydrate $\left(\mathrm{HOC}(\mathrm{COONa})\left(\mathrm{CH}_{2} \mathrm{COONa}\right)_{2} \cdot 2 \mathrm{H}_{2} \mathrm{O}\right)$, ATP, tris (2-carboxyethyl) phosphine hydrochloride (TCEP), polyvinylpyrrolidone (PVP), sodium chloride $(\mathrm{NaCl})$, ethyl alcohol, phosphate-buffered saline solution ( $\mathrm{pH} 7.4,1 \mathrm{M})$, Tris-EDTA buffer solution (TE, 100X), and saline-sodium citrate buffer (SSC, $\mathrm{pH} 7.0,20 \times$ ) were purchased from Sigma-Aldrich (St. Louis, MO, USA). MGITC was purchased from Life Technologies (Eugene, OR, USA). Streptavidin-activated magnetic beads (Dynabeads MyOne) were purchased from Invitrogen (Beaverton, OR, USA). Formvar/carbon coated TEM grids (200 mesh, 01800-F) were purchased from TED PELLA, Inc. (Redding, CA, USA). Capture, target, and gene marker DNA oligonucleotides were purchased from Integrated DNA Technologies, Inc. (Coralville, IA, USA). Ultrapure deionized water obtained using the arium comfort Lab Water System (Sartorius, Goettingen, Germany) was used in all experiments.

Instrumentation: The SEM images were obtained using a JSM-7800F Prime instrument (JEOL Ltd., Japan) at an accelerating voltage of $5 \mathrm{kV}$. High-magnification TEM images and EDS element mapping images were obtained using a JEM-F200 instrument (JEOL Ltd., Japan) at an accelerating voltage of $200 \mathrm{kV}$. The DLS data were acquired using a Nano-ZS90 apparatus (Malvern Instruments, Malvern, UK). UV-vis absorption spectra were obtained using a Cary 100 spectrophotometer (Varian, Salt Lake City, USA) and a Synergy H1 Hybrid multi-mode reader (BioTek, Winooski, VT, USA). SERS mapping images were obtained using an inVia Raman microscope system (Renishaw, New Mills, UK); a He-Ne laser operating at $\lambda=632.8 \mathrm{~nm}$ was utilized as an excitation source. The Raman scattering signals were collected 
using a charge-coupled device camera with a high-resolution grating (1200 grooves $\mathrm{mm}^{-1}$ ), and measured by focusing a laser spot using a 20x (NA 0.4) objective lens. The baseline correction of each Raman spectrum was performed using the Renishaw WIRE 5.3 software.

Synthesis of AuNPs: AuNPs (20 nm in size) were prepared using the Frens method. ${ }^{[26]}$ Briefly, $50 \mathrm{~mL}$ of $0.01 \%(\mathrm{w} / \mathrm{w}) \mathrm{HAuCl}_{4}$ was added to a two-necked round-bottomed flask and heated under vigorous stirring. Sodium citrate $[1 \mathrm{~mL}$ of $1 \%(\mathrm{w} / \mathrm{w})]$ was added to the flask when the solution started to boil. The color of the solution changed from deep purple to red after $30 \mathrm{~min}$ and the resulting solution was stirred for $1 \mathrm{~h}$ at room temperature. Other AuNPs with larger sizes (40,50, and $80 \mathrm{~nm}$ ) were prepared by slightly modifying a previously reported seed growth synthesis method. ${ }^{[27]} \mathrm{A} 2.2 \times 10^{-3} \mathrm{M}$ sodium citrate solution was heated with rapid stirring in a three-neck round-bottomed flask. Upon boiling, $0.5 \mathrm{~mL}$ of $25 \times 10^{-3} \mathrm{M} \mathrm{HAuCl}_{4}$ was added to the flask to form Au seeds. The solution was cooled in the same flask until the temperature reached $90^{\circ} \mathrm{C}$. At that moment, $0.5 \mathrm{~mL}$ of $25 \times 10^{-3} \mathrm{M}$ $\mathrm{HAuCl}_{4}$ was added to the flask, and the reaction was completed after $30 \mathrm{~min}$. This process was repeated twice. Subsequently, the solution was diluted by adding $26.5 \mathrm{~mL}$ of DW and $1 \mathrm{~mL}$ of $60 \mathrm{~mm}$ sodium citrate to $27.5 \mathrm{~mL}$ of the extracted solution. This process was repeated three times.

Fabrication of Dimpled Au Substrates: A $125 \mu$ m-thick PEN, purchased from Dupont, was used after removing its protective film. The polymer film was treated with linear $\mathrm{O}_{2}$ ion beam bombardment in a linear moving state, which resulted in a nanodimpled pattern. ${ }^{[28,29]}$ The inlet $\mathrm{O}_{2}$ flow rate and the working pressure were fixed at $70 \mathrm{sccm}$ and $0.9 \mathrm{mTorr}$, respectively, during the $\mathrm{O}_{2}$ ion beam treatment. The PEN substrates were subjected to 40 scans in a reciprocating motion at a linear moving speed of $10 \mathrm{~mm} \mathrm{~s}^{-1}$, which corresponded to an $\mathrm{O}_{2}$ ion irradiation dose of $9.4 \times 10^{16} \mathrm{~cm}^{-2}$. The mean ion energy was measured to be $700 \pm 70 \mathrm{eV}$ using an ion energy analyzer. A $150 \mathrm{~nm}$-thick $\mathrm{Au}$ layer was directly deposited onto the PEN nanodimples at a deposition rate of $2.0 \AA \mathrm{s}^{-1}$ using a thermal evaporation system (LAT, Korea). The base pressure of the chamber was set to $9.6 \times 10^{-6}$ Torr.

Preparation of DNA-Conjugated AuNPs: The detection DNAs were immobilized on the AuNP surfaces to prepare DNA-conjugated AuNPs. Thiol-modified DNA (5'-TTC TCC TCA ATT TTT TTT TT- $\left.\left(\mathrm{CH}_{2}\right)_{3}-\mathrm{HS}-3^{\prime}\right)$ was prepared using DNA in the disulfide form by the following method. First, $1 \mu \mathrm{L}$ of $100 \mu \mathrm{M}$ disulfide DNA was mixed with $1 \mu \mathrm{L}$ of $5 \mathrm{~mm}$ TCEP. The mixture was reacted for $30 \mathrm{~min}$ at room temperature to produce free thiol groups. The mixture was diluted with $1 \times T E$ after the reaction. A DNA conjugation reaction was performed overnight at $4{ }^{\circ} \mathrm{C}$ after adding the thiol-modified DNAs to $1 \mathrm{~mL}$ of AuNPs. For stabilization, $50 \mu \mathrm{L}$ of $15 \%$ PVP in $0.2 \mathrm{M}$ PB was added to the DNA-AuNP mixture and stirred for $30 \mathrm{~min}$; subsequently, $40 \mu \mathrm{L}$ of $5 \mathrm{M} \mathrm{NaCl}$ was reacted with this mixture overnight at $4{ }^{\circ} \mathrm{C}$. The resulting mixture was centrifuged at a relative centrifugal force of 1000 for 40 min to remove excess reagents. The DNA-conjugated AuNPs were restored in 1× TE after removing the supernatant solution.

Internalization of AuNPs in the Cavities of the Dimpled Au Substrate: AuNPs were internalized in the nanocavities of the dimpled Au substrate by two different methods (electrostatic interactions between AuNPs and ATP, and DNA hybridization of AuNPs and nanocavities). Two different Au substrates (2D flat and nanodimples) were cut to a size of $5 \times 5 \mathrm{~mm}^{2}$ and washed with ethanol. Each substrate was dipped into a microtube with $10^{-3} \mathrm{M}$ ATP for $4 \mathrm{~h}$. After washing with ethanol and DW three times, $10 \mu \mathrm{L}$ of $0.1 \mathrm{~nm}$ AuNPs was dropped onto each substrate for $1 \mathrm{~h}$ in a humid chamber. The resulting nanodimpled Au substrate was cleaned with ethanol and $1 \times$ TE. To transform disulfide DNA to thiol DNA (5"-Cy5-TTG AGG AGA ATT TTT TTT TT- $\left.\left(\mathrm{CH}_{2}\right)_{3}-\mathrm{HS}-3^{\prime \prime}\right), 20 \mu \mathrm{L}$ of $100 \mu \mathrm{M}$ disulfide DNA was reacted with $20 \mu \mathrm{L}$ of $5 \mathrm{~mm}$ TCEP for $30 \mathrm{~min}$ at room temperature. To improve the efficiency of immobilization of the capture DNA on the dimpled Au surface, $5 \mathrm{M} \mathrm{NaCl}$ in a $1 \times \mathrm{TE}$ solution was added to the mixture. The substrate was subsequently incubated with capture DNAs overnight at $37{ }^{\circ} \mathrm{C}$. After immobilization, the substrate was washed with $2 \times$ SSC and subsequently reacted with detection-DNA-conjugated AuNPs with $2 \times$ SSC for $1 \mathrm{~h}$ at $37^{\circ} \mathrm{C}$ in a humid chamber. The substrate was then rinsed twice with $0.5 \times$ SSC. Unbound DNAs were removed from the substrate using ultrasonication.

SERS-Based Assays of COVID-19 Target Genes: DNA assays based on the AuNP-internalized nanodimpled Au substrate and AuNP-hybridized magnetic beads were performed to evaluate the sensitivity of the SERSbased assay platform designed in this study. Herein, the COVID-19 genes, RdRp and $\mathrm{E}$, were used as target DNA markers. The RdRpcapture DNA probe $\left(5^{\prime}-\right.$ ACGTTCCACCTCTTTTTTTT- $\left(\mathrm{CH}_{2}\right)_{3}$-biotin/ $\left.\mathrm{SH}-3^{\prime}\right)$ and E-gene-capture DNA probe $\left(5^{\prime}-\mathrm{GATGGCTAGTCTTTTTTTT}\right.$ $\mathrm{T}-\left(\mathrm{CH}_{2}\right)_{3}$-biotin/ $\left.\mathrm{SH}-3^{\prime}\right)$ were immobilized on the surface of magnetic beads and in the cavities of the nanodimpled Au substrate, respectively. In the magnetic-bead-based DNA assays, $5 \mu \mathrm{L}$ of the corresponding COVID-19 gene marker was reacted with $75 \mu \mathrm{L}$ of $0.12 \mathrm{~nm}$ detectionDNA-conjugated SERS nanotags (40 nm in diameter) and $10 \mu \mathrm{L}$ of 1.6 pM capture-DNA-immobilized magnetic beads for the formation of DNA-sandwich complexes. Subsequently, $10 \mu \mathrm{L}$ of a $20 \times$ SSC buffer solution was added to prevent steric effects. After $40 \mathrm{~min}$ of the reaction, the magnetic DNA-sandwich complexes were washed with $1 \times$ SSC buffer, separated from the supernatant solution using a magnet, and subsequently resuspended in a 1X TE buffer for the SERS experiments. In the assays based on AuNP-internalized nanodimpled Au substrates, a solution containing $5 \mu \mathrm{L}$ of the COVID-19 gene marker, $30 \mu \mathrm{L}$ of $0.05 \mathrm{~nm}$ detection-DNA-conjugated SERS nanotags ( $80 \mathrm{~nm}$ in diameter), and $5 \mu \mathrm{L}$ of a $20 \times$ SSC buffer was dropped on the surface of the captureDNA-immobilized nanodimpled $\mathrm{Au}$ substrate, followed by incubation for $40 \mathrm{~min}$ in a humid chamber at $50^{\circ} \mathrm{C}$. The DNA-sandwich complexes were washed with $1 \times$ SSC buffer and water, and subsequently dried in an oven at $70^{\circ} \mathrm{C}$ for the SERS experiments.

FDTD Simulations: 3D FDTD simulations were performed using commercial software (Lumerical, Ansys) to investigate the distribution of the electromagnetic (EM) field generated on the SERS substrates. To calculate the EM field of the $80 \mathrm{~nm}$-sized AuNP-internalized nanodimpled Au substrate, the curvature of the bottom of the cavity of the nanodimpled Au substrate was set to $32.4 \mu \mathrm{m}^{-1}$, and the top radius and depth were set to 104 and $166 \mathrm{~nm}$, respectively. In the ATP-based method, the nanogap distance between the AuNPs and the curved surface of the cavities, and the refractive index were set to $1 \mathrm{~nm}$ and 1.663 , respectively. Furthermore, the nanogap and refractive index were set to $4 \mathrm{~nm}$ and 1.462, respectively, in DNA hybridization. The simulation was performed in a space of $0.6 \mu \mathrm{m}$ for the $x$ - and $\gamma$-axes and $0.8 \mu \mathrm{m}$ for the $z$-axis; the boundary condition involved assuming perfectly matched layers. The size of the Yee cell was set to $0.5 \mathrm{~nm}$ for the $x_{-}, y$-, and $z$-axes. A $633 \mathrm{~nm}$-wavelength plane wave polarized in the $x$-direction was employed as the light source and incident on the structure along the $z$-direction. ${ }^{[30-32]}$

\section{Supporting Information}

Supporting Information is available from the Wiley Online Library or from the author.

\section{Acknowledgements}

H.D. and S.-G.P. contributed equally to this work. This study was supported by the National Research Foundation of Korea (grant numbers 2019R1A2C3004375 and 2020R1A5A1018052), the Fundamental Research Program (PNK 7440) of the Korean Institute of Materials Science (KIMS), and the MOTIE (20012405). National Nature Science Foundation of China (grant number 21976209) and Taishan Scholar Project Special Funding (No. ts20190962) also supported this work.

\section{Conflict of Interest}

The authors declare no conflict of interest. 


\section{Data Availability Statement}

Research data are not shared.

\section{Keywords}

electromagnetic enhancement, nanodimpled substrate, nanogaps, plasmonic coupling, surface-enhanced Raman scattering

Received: June 14, 2021

Revised: July 14, 2021

Published online:

[1] J. J. Baumberg, J. Aizpurua, M. H. Mikkelsen, D. R. Smith, Nat. Mater. 2019, 18, 668.

[2] S. Chen, Y. Zhang, T.-M. Shih, W. Yang, S. Hu, X. Hu, J. Li, B. Ren, B. Mao, Z. Yang, Z. Tian, Nano Lett. 2018, 18, 2209.

[3] Z. Wang, S. Zong, L. Wu, D. Zhu, Y. Cui, Chem. Rev. 2017, 117, 7910.

[4] K. Wu, T. Li, M. S. Schmidt, T. Rindzevicius, A. Boisen, S. Ndoni, Adv. Funct. Mater. 2018, 28, 1704818.

[5] B. Liu, X. Yao, S. Chen, H. Lin, Z. Yang, S. Liu, B. Ren, Adv. Funct. Mater. 2018, 28, 1802263.

[6] Z. Lou, L. Wang, K. Jiang, G. Shen, Nano Today 2019, 26, 176.

[7] M. J. Urban, C. Shen, X.-T. Kong, C. Zhu, A. O. Govorov, Q. Wang, M. Hentschel, N. Liu, Annu. Rev. Phys. Chem. 2019, 70, 275.

[8] S. -W. Hsu, A. L. Rodarte, M. Som, G. Arya, A. R. Tao, Chem. Rev. 2018, 118, 3100.

[9] D. Luo, C. Yan, T. Wang, Small 2015, 11, 5984.

[10] D. Graham, D. G. Thompson, W. E. Smith, K. Faulds, Nat. Nanotechnol. 2008, 3, 548.

[11] H.-H. Jeong, M. C. Adams, J.-P. Gunther, M. Alarcon-Correa, I. Kim, E. Choi, C. Miksch, A. F. Mark, A. G. Mark, P. Fischer, ACS Nano 2019, 13, 11453.

[12] J. Li, T.-S. Deng, X. Liu, J. A. Dolan, N. F. Scherer, P. F. Nealey, Nano Lett. 2019, 19, 4314.
[13] K. Tapio, A. Mostafa, Y. Kanehira, A. Suma, A. Dutta, I. Bald, ACS Nano 2021, 15, 7065

[14] L. Guerrini, D. Graham, Chem. Soc. Rev. 2012, 41, 7085.

[15] N. N. Nam, T. L. Bui, S. J. Son, S.-W. Joo, Adv. Funct. Mater. 2019, 29, 1809146.

[16] S.-G. Park, X. Xiao, J. Min, C. W. Mun, H. S. Jung, V. Giannini, R. Weisslder, S. A. Maier, H. Im, D.-H. Kim, Adv. Funct. Mater. 2019, 29, 1904257.

[17] S.-G. Park, C. W. Mun, X. Xiao, A. Braun, S. Kim, V. Giannini, S. A. Maier, D.-H. Kim, Adv. Funct. Mater. 2017, 27, 1703376.

[18] X. He, E. H. Y. Lau, P. Wu, X. Deng, J. Wang, X. Hao, Y. C. Lau, J. Y. Wong, Y. Guan, X. Tan, X. Mo, Y. Chen, B. Liao, W. Chen, F. Hu, Q. Zhang, M. Zhong, Y. Wu, L. Zhao, F. Zhang, B. J. Cowling, F. Li, G. M. Leung, Nat. Med. 2020, 26, 672.

[19] L. Ferretti, C. Wymant, M. Kendall, L. Zhao, A. Nurtay, L. AbelerDörner, M. Parker, D. Bonsall, C. Fraser, Science 2020, 368, eabb6936.

[20] M. Shen, Y. Zhou, J. Ye, A. A. Abdullah AL-Maskri, Y. Kang, S. Zeng, S. Cai, J. Pharm. Anal. 2020, 10, 97.

[21] Y. Wu, N. Choi, H. Chen, H. Dang, L. Chen, J. Choo, Anal. Chem. 2020, 92, 2628.

[22] F. M. Huang, D. Wilding, J. D. Speed, A. E. Russell, P. N. Bartlett, J. J. Baumberg, Nano Lett. 2011, 11, 1221.

[23] R. Chikkaraddy, V. A. Turek, N. Kongsuwan, F. Benz, C. Carnegie, T. van de Goor, B. de Nijs, A. Demetriadou, O. Hess, U. F. Keyser, J. J. Baumberg, Nano Lett. 2018, 18, 405.

[24] H. Wei, H. Xu, Nanoscale 2013, 5, 10794.

[25] D.-K. Lim, K.-S. Jeon, H. M. Kim, J.-M. Nam, Y. D. Suh, Nat. Mater. 2010, 9, 60 .

[26] G. Frens, Nat. Phys. Sci. 1973, 241, 20.

[27] N. G. Bastus, J. Comenge, V. Puntes, Langmuir 2011, 27, 11098.

[28] S. Lee, E. Y. Byun, J. K. Kim, D. G. Kim, Curr. Appl. Phys. 2014, 14, S180.

[29] H. Chen, S.-G. Park, N. Choi, J. I. Moon, H. Dang, A. Das, S. Lee, D. G. Kim, L. Chen, J. Choo, Biosens. Bioelectron. 2020, 167, 112496.

[30] Y. Mantri, J. V. Jokerst, ACS Nano 2020, 14, 9408.

[31] L. Liu, R. Aleisa, Y. Zhang, J. Feng, Y. Zheng, Y. Yin, W. Wang, Angew. Chem., Int. Ed. 2019, 58, 16307.

[32] C. Cherqui, M. R. Bourgeois, D. Wang, G. C. Schatz, Acc. Chem. Res. 2019, 52, 2548. 